AperTO - Archivio Istituzionale Open Access dell'Università di Torino

Clinicopathological study of a series of 92 adrenocortical carcinomas: from a proposal of simplified diagnostic algorithm to prognostic stratification.

This is a pre print version of the following article:

Original Citation:

Availability:

This version is available http://hdl.handle.net/2318/68505

since 2016-06-11T12:16:18Z

Published version:

DOI:10.1111/j.1365-2559.2009.03423.x

Terms of use:

Open Access

Anyone can freely access the full text of works made available as "Open Access". Works made available under a Creative Commons license can be used according to the terms and conditions of said license. Use of all other works requires consent of the right holder (author or publisher) if not exempted from copyright protection by the applicable law. 


\section{Histopathology}

\section{Clinico-pathological study of a series of 92 adrenocortical carcinomas: from a proposal of simplified diagnostic algorithm to prognostic stratification}

\begin{tabular}{|r|l|}
\hline Journal: & Histopathology \\
\hline Manuscript ID: & draft \\
\hline Manuscript Type: & Original Article \\
\hline Complete List of Authors: & $\begin{array}{l}\text { Volante, Marco; University of Turin, Department of Clinical and } \\
\text { Biological Sciences, Division of Pathology } \\
\text { Bollito, Enrico; San LUigi Hospital, Pathology } \\
\text { Sperone, Paola; San Luigi Hospital, Oncology } \\
\text { Tavaglione, Veronica; University of Turin, Department of Clinical } \\
\text { and Biological Sciences, Division of Pathology } \\
\text { Daffara, Fulvia; San Luigi Hospital, Internal Medicine } \\
\text { Porpiglia, Francesco; University of Turin, Department of Clinical and } \\
\text { Biological Sciences, Division of Urology } \\
\text { Terzolo, Massimo; University of Turin, Department of Clinical and } \\
\text { Biological Sciences, Division of Internal Medicine } \\
\text { Berruti, Alfredo; University of Turin, Department of Clinical and } \\
\text { Biological Sciences, Division of Oncology } \\
\text { Papotti, Mauro; University of Turin, Department of Clinical and } \\
\text { Biological Sciences, Division of Pathology }\end{array}$ \\
\hline Keywords: & \begin{tabular}{l} 
Adrenal cortex, carcinoma, diagnosis, prognosis, reticulin stain \\
\hline \hline
\end{tabular} \\
\hline \hline
\end{tabular}

\section{S) ScholaroNE \\ Manuscript Central}




\title{
Clinico-pathological study of a series of 92 adrenocortical carcinomas: from a proposal of simplified diagnostic algorithm to prognostic stratification
}

\author{
Marco Volante ${ }^{1}$, Enrico Bollito ${ }^{1}$, Paola Sperone ${ }^{2}$, Veronica Tavaglione ${ }^{1}$, Fulvia Daffara ${ }^{3}$, \\ Francesco Porpiglia ${ }^{4}$, Massimo Terzolo ${ }^{3}$, Alfredo Berruti ${ }^{2}$, Mauro Papotti ${ }^{1}$ \\ ${ }^{1}$ Divisions of Pathology, ${ }^{2}$ Oncology, ${ }^{3}$ Internal Medicine and ${ }^{4}$ Urology, Department of Clinical \\ and Biological Sciences, University of Turin at San Luigi Hospital, Turin, Italy.
}

\section{Corresponding Author:}

Marco Volante MD

Department of Clinical and Biological Sciences, University of Turin at San Luigi Hospital, Regione Gonzole 10, 10043 Orbassano, Turin, Italy

Phone: +390116705464; Fax +390116705432 E-Mail: marco.volante@unito.it

Running title: Algorithmic approach to adrenal cancer

Keywords: Adrenal cortex, carcinoma, diagnosis, prognosis, reticulin stain 


\section{Abstract}

Aim. Pathological diagnosis of adrenocortical carcinoma relies on several microscopic features commonly used in combination within different scoring systems that are sometimes subjective and/or time consuming. Aim of our study was to investigate the impact of individual pathological parameters in the diagnosis and prognosis of adrenocortical carcinoma. Methods and Results. The series included 92 malignant cases and a control series of 47 adenomas, all classified according to the Weiss score criteria. The presence of the disruption of the reticular network, as highlighted by reticulin histochemical staining, was present in all adrenocortical carcinomas and the inclusion of at least one of the three following additional parameters - mitotic index $>5 / 50 \mathrm{HPF}$, presence of necrosis and presence of vascular invasion - designed an algorithm with a $100 \%$ sensitivity and specificity to recognize malignant tumors according to the Weiss system, with an easier and more practical applicability. Moreover, by multivariate analysis, stage III/IV and mitotic count $>9 / 50 \mathrm{HPF}$ showed a strong adverse impact on disease-free and overall survival, leading to the identification of three risk groups affected by a significantly different prognosis. Conclusions. The present paper defines an easy-to-perform and highly specific and sensitive algorithm for the diagnosis and prognostic categorization of malignant adrenocortical tumors. 


\section{Introduction}

Adrenocortical carcinoma is a rare tumor of the adrenal cortex which accounts for no more than $0.2 \%$ of all malignancies, with two peaks of incidence in early childhood and adults over age 60 . It represents an aggressive disease with up to two thirds of cases having distant metastases at the time of diagnosis and a mean survival of less than 30 months, ${ }^{1-3}$ although combined treatment modalities, with special reference to mitotane-based therapy, seems to improve its outcome. ${ }^{4}$

The differential diagnosis of carcinomas from adenomas has been based on several microscopic features, none of them being alone absolutely indicative of malignancy. ${ }^{5,6}$ Despite the majority of adrenocortical carcinomas do not represent a diagnostic pitfall in the clinical practice, there are several other cases in which the distinction from their benign counterparts is not straightforward. These cases are a challenge for the pathologist, since the therapeutic strategy in adrenocortical carcinomas is radically different from that of adenomas and an accurate diagnosis is mandatory. This issue is also relevant because adrenal tumors are increasingly recognized in clinical practice: the widespread use of imaging techniques has resulted in the clinical dilemma of the adrenal incidentaloma, ${ }^{7}$ and although the vast majority of adrenal incidentalomas are benign lesions, in up to $12 \%$ of cases an incidental adrenocortical carcinoma may be discovered. ${ }^{8}$

Different scoring systems for adrenocortical carcinoma have therefore been developed, using mathematical models or numerical scores based on the association of a given threshold for each considered parameter to malignancy. ${ }^{9-11}$ The most widely employed scoring system was proposed ${ }^{11}$ and revisited in a subsequent report ${ }^{12}$ by Weiss, and includes nine parameters related to tumor structure (loss of clear cytoplasm, presence of diffuse architecture and of necrosis), cytological features (atypia, mitotic count, atypical mitotic figures) and invasive properties (sinusoidal, venous and capsular invasion).

Unfortunately, Weiss and other scoring systems are sometimes difficult to apply, subjective and/or time consuming, despite several re-visitations and implementations occurred along years to 
simplify the diagnostic procedure and provide clinically relevant information. ${ }^{13-15}$ Moreover, in paediatric adrenocortical tumors some features included in the Weiss system (namely eosinophilic cytoplasm, diffuse architecture or sinusoidal invasion) were found to be less relevant as compared to adult tumors, ${ }^{16}$ thus indicating that the diagnostic work up of children's tumors should probably employ a different panel of pathological factors.

Recently, a different approach based on the definition of an algorithm centred on mitotic count $(>5 / 50 \mathrm{HPF})$, followed by the recognition of other parameters (including nuclear grade, diffuse growth pattern and tissue reaction) was proposed, ${ }^{17}$ and although based on a relatively small series of malignant cases - defined by the presence of metastases - it had the advantage of simplifying the current diagnostic approach based on numerical scores.

A second major issue is the distinction between morphologically and clinically malignant adrenocortical tumors. Not all adrenal tumors having unequivocal microscopic features of malignancy progress to fatal outcome, therefore a better definition of the clinical and pathological features that impact on prognosis and a stratification of adrenal cancer patients into prognostic categories are needed. Among the most commonly used pathological parameters for the diagnosis of adrenocortical carcinoma, mitotic activity and/or proliferative index showed a significant association with survival, ${ }^{10,12,18}$ as age and overall Weiss score did, as well. ${ }^{19}$ In a very large study on metastatic tumors aimed to recognize prognostic rather than diagnostic parameters, multivariate analysis identified the presence of a mitotic rate $>20 \times 50$ HPF and the number of organs involved as the major factors that influence prognosis in advanced adrenal cancer patients. ${ }^{20}$

The aim of the present study was to reconsider the clinical pathological parameters currently used in the diagnosis of adrenocortical carcinoma in a large retrospective series of 92 cases diagnosed and treated at one Institution, with the aim to revise the classical diagnostic scoring systems in an algorithmic approach and to provide a pathologically-based prognostic stratification of adrenal cancer patients. 


\section{Materials and methods}

\section{Case selection and pathological classification.}

From a total of 153 cases of primary adrenocortical carcinomas of an adult population included in the clinical databases (from 1995 to 2007) of the Divisions of Oncology and Internal Medicine at San Luigi Hospital, University of Turin, that serves as national referral centre for this neoplasm, 92 cases had pathological material retrievable from the files of the Pathology Departments of the University of Turin (including 44 consultation cases, operated elsewhere). All cases were independently classified by three of us (MV, EB, MP) re-assessing the parameters commonly considered in the Weiss system, using 2 to 10 slides for each case. All parameters included in the Weiss system ${ }^{12}$ were recorded independently, together with clinical pathological information such as sex, age, location, clinical syndrome, weight and size. For comparison, all cases were also scored using the Weiss modified (according to Aubert et al.) ${ }^{14}$ and Van Slooten ${ }^{10}$ scoring systems. Baseline demographic and clinical data were complete and follow up information were available for survival analysis in all but 15 cases, either recent (7 cases) or lost at follow up (8 cases). Tumor staging was assessed following recommendations by Sullivan et al. and the Armed Force Institute of Pathology. ${ }^{21,22}$

Seventy-nine patients bearing stage I to III disease underwent radical surgery followed by adjuvant mitotane in 14 of them. The remaining 13 patients, that were not amenable to surgery due to locally advanced or metastatic disease, received Mitotane alone (2 patients) or Mitotane plus chemotherapy (11 patients) as upfront treatment. One of them was radically operated after 6 cycles of chemotherapy plus Mitotane.

Additional 47 adrenocortical tumors, including all cases observed in the same time period having Weiss scores 1 or 2 (16 cases) or adenomas with large size, were collected as a benign tumor control group. 
The main clinical and pathological features of the whole series of 139 adrenocortical tumors are summarized in Table 1.

\section{Histochemistry and immunohistochemistry.}

In selected cases, to confirm the adrenocortical nature of the lesion, immunohistochemical staining for chromogranin A (Novocastra, Newcastle, UK; clone LK2H10, diluted 1:800), synaptophysin (DakoCytomation, Glostrup, DK; clone SY38, diluted 1:100), Melan A (DakoCytomation; clone A103, diluted 1:50), alpha-inhibin (Diamedix, Miami, Florida; clone R1, diluted 1:75) and CD10 (Santa Cruz Biotech., Santa Cruz, CA; clone 56C6, diluted 1:30) were performed. A standard automated (Dako Autostainer, Glostrup, Denmark) immunoperoxidase procedure was employed, and immunoreactions were revealed by a biotin-free dextran-chain detection system (Envision, Dako), and developed using diaminobenzidine as the chromogen.

To better evaluate the growth pattern, which is sometimes hard to assess on pure haematoxylin and eosin staining, in all cases of adrenal carcinoma with a non-diffuse growth according to the original definition of Weiss ${ }^{12}$ and in all adrenocortical adenomas, a reticulin histochemical staining was performed to define the status of the reticulin framework, using a commercially available silver impregnation-based kit (Bio Optica, Milan, Italy). Moreover, basal membrane material was highlighted by immunohistochemical detection of laminin (Novocastra; clone 4C7, diluted 1:50) and collagen type IV (DakoCytomation; clone CIV22, diluted 1:100), as detailed above. The presence of a disruption of the reticulin/basal membrane network, similar to that described in the setting of other endocrine diseases ${ }^{23,24}$ was defined by the loss of continuity of reticular fibres or basal membrane network evaluated in one high power field (400x), extended to at least one third of the lesion. 


\section{Statistical analysis.}

All data were analyzed with STATISTICA for Windows software version 6.1 (Stat Soft, Italy). A level of $\mathrm{p}<0.05$ was considered statistically significant.

Distribution of single Weiss's criteria in the overall group of 139 adrenocortical neoplasms was evaluated using contingency tables to calculate sensitivity and specificity in relation to the diagnosis of adrenocortical carcinoma. To analyze the prognostic impact of all clinical pathological variables considered, univariate disease free and overall survival analysis was based on the Kaplan-Meier product limit estimate of survival distribution. Clinical pathological parameters considered in survival analysis of adrenocortical carcinoma group included: sex, age, location, size, weight, stage, Weiss, Weiss modified and van Slooten overall scores, and all single nine histological parameters considered in the Weiss scoring system. Unadjusted differences between survival curves were tested using the LogRank test. Multivariate analysis of the relative impact on survival of each parameter included in the univariate analysis was estimated using the Cox proportional hazards regression model.

\section{Results}

\section{Distribution of the Weiss's parameters in adrenocortical tumors.}

The summary of the Weiss's parameter(s) distribution in the whole series of 139 adrenocortical neoplasms is reported in Table 2. None of the parameters reached both $100 \%$ sensitivity and specificity for the diagnosis of adrenocortical carcinoma. All but three parameters (presence of diffuse architecture, nuclear atypia and eosinophilic cytoplasm) were $100 \%$ specific for a diagnosis of adrenocortical carcinoma. The most sensitive feature was necrosis, that was recognizable in $84 \%$ of cases, followed by mitotic count exceeding 5 in 50 HPF (71\%) and venous invasion (64\%). The presence of atypical mitoses was highly specific but it was observed in $34 \%$ of cases, only. 


\section{Definition of disrupted reticulin framework.}

A diffuse growth pattern was detected in $79 \%$ of adrenocortical carcinomas, and in $9 \%$ of adrenocortical tumors having a Weiss score $<3$. Diffuse architecture was considered according to Weiss ${ }^{12}$ as the presence of patternless sheets of cells in more than one-third of the tumor, being trabecular, cordonal, columnar, alveolar, or nesting organizations considered as non-diffuse patterns. In order to better define the pattern of growth, in all adrenocortical carcinoma cases with a non-diffuse architecture at histology, as well as in all 47 adrenocortical adenoma samples, histochemical staining for reticulin and immunohistochemical staining with laminin and/or collagen IV were performed. A disruption of the reticulin/basal membrane network was detected in all adrenocortical carcinoma samples (usually in more than half of the tumor) (Figure 1). In adrenocortical tumors with a borderline Weiss value (score 2), two cases only, having a diffuse and trabecular growth rather than the alveolar architecture typical of adrenocortical adenoma, showed the loss of a regularly distributed reticulin network. Both such tumors had large size $(9$ and $6 \mathrm{~cm})$ and in addition to a diffuse pattern had either nuclear atypia or eosinophilic cytoplasm, and are alive and well at 2 and 3 years follow up in the absence of any adjuvant therapy after surgery. Indeed, the morphological (Weiss definition of diffuse growth) and/or histochemical/immunohistochemical demonstration of the lack of a well preserved reticulin framework was $100 \%$ sensitive and $96 \%$ specific in the identification of malignant adrenocortical lesions (Weiss score $\geq 3$ ).

\section{Design of a simplified diagnostic algorithm.}

The analysis of the frequency of any single Weiss's parameter in this large series of adrenocortical tumors led to design a diagnostic algorithm based on the most sensitive and specific features, replacing the classical numerical scoring systems.

The algorithm takes into consideration the presence of a disrupted reticulin framework, that was the single most sensitive feature of malignancy, followed by at least one of the three parameters with $100 \%$ specificity and highest sensitivity and specificity, namely mitotic count $>5 \times 50 \mathrm{HPF}$, 
presence of necrosis (irrespective of its extent) and/or venous invasion (Figure 2). The retrospective evaluation of the whole series of 139 adrenocortical lesion classified according to the Weiss system demonstrated that this algorithm was $100 \%$ sensitive and specific for recognizing all cases having a Weiss score $\geq 3$, thus considered malignant.

\section{Prognostic stratification of adrenocortical carcinoma patients.}

A secondary aim of the present study was the prognostic stratification of adrenocortical cancer patients. Among the clinical and pathological parameters investigated by means of univariate analysis, advanced stage (stage 3 and 4; $=0.007$ ), mitotic count higher than the median value of 9 $(p=0.02)$, presence of atypical mitoses $(p=0.047)$, Weiss score higher than $6(p=0.02)$, and Weiss modified score higher than $5(\mathrm{p}=0.02)$ were significantly associated to a worse overall survival, whereas age over the median of 45 years $(p=0.04)$, stage (stage 3 and $4 ; p=0.008)$ and mitotic count over the median value of $9(\mathrm{p}=0.04)$ were associated to a shorter disease free survival.

Multivariate Cox regression model showed an independent prognostic role, at both disease free and overall survival analysis, for stage, mitotic count and presence of diffuse growth pattern (Table 3).

Based on multivariate analysis, adrenocortical carcinomas were re-classified into three risk groups according to the status of the independent prognostic factors (diffuse growth pattern was excluded, being part of the diagnostic algorithm as the first step criterion for adrenal carcinoma). Survival analysis demonstrated that the stratification of adrenocortical cancer patients according to the scheme proposed was strongly correlated to disease free $(p<0.007)$ and overall $(p<0.005)$ outcome status (Figure 3). Mean disease-free survival were 61.9, 17.3, 12.2 months, and overall survival were not attained, 66.2, 26.4 months in low, intermediate and high risk groups, respectively. 


\section{Discussion}

In the present paper, a novel diagnostic approach and a prognostic stratification of adrenal cancer was defined by extensive analysis of clinical pathological features in a large series of tumors. The major relevant findings of our study were i) the definition of the presence of disrupted reticulin framework as an hallmark for the diagnosis of adrenocortical carcinoma; ii) the proposal of a simplified diagnostic algorithm based on the recognition of unequivocal malignancy-related features rather than on numerical scores; iii) the prognostic categorization of adrenal cancer patients into three groups characterized by significantly different outcome.

Our first observation stemmed by the re-evaluation of the concept of diffuse growth pattern, that in both Weiss' original papers ${ }^{11,12}$ and also in a recent study ${ }^{17}$ was highlighted as a major diagnostic feature for adrenal cancer, although less relevant for others. ${ }^{14}$ By morphological analysis and appropriate histochemical and immunohistochemical reactions, all adrenocortical carcinomas were found to have a disruption of the reticulin framework, irrespective of the growth pattern. In other terms, not only diffuse growth but also trabecular, cordonal, nesting or other patterns were affected by the loss of the reticulin/basal membrane network typical of normal or adenomatous adrenal cortex, thus reassessing the concept of growth pattern as a primary feature in adrenocortical carcinoma diagnosis. Interestingly, such observation fits with some recent findings from our group on the expression by adrenal cancer cells of proteins involved in matrix digestion, such as matrixmetalloproteinase type $2,{ }^{25}$ and on the de-regulation of gene transcription of laminin isoforms in malignant as compared to non neoplastic adrenal tissue (Volante M et al, submitted).

From a diagnostic point of view, the presence of a disrupted reticulin network was a highly sensitive and specific feature in adrenocortical carcinoma diagnosis. Since its recognition is easy and equivocal cases might be solved by reticulin staining and/or laminin and type IV collagen immunohistochemistry, we entered this criterion as the primary step of the diagnostic algorithm herein proposed. The additional presence of at least one of the malignancy-related features conventionally considered in several endocrine tumors (necrosis, high mitotic rate and venous 
vessel invasion) raised up to $100 \%$ the sensitivity and specificity of the algorithm for a diagnosis of malignancy. The decision of proposing a two-step algorithm, including at a second level additional microscopic features of malignancy, was related to the finding of disruption of the reticulin framework in two cases of large size adenomas, which reached a Weiss score 2: although clinical data were consistent in both cases with a benign disease at the last follow up, the biological meaning of the observed reticulin alteration remains to be defined.

This simplified scheme has the major advantage of reducing the analysis of other parameters such as nuclear atypia, eosinophilic cytoplasm, sinusoidal and capsular invasion, that although commonly found in malignant lesions may be of equivocal definition and are sometimes subjective, especially in pathology laboratories lacking a specific experience in endocrine diseases. It is important to underline that in the great majority of cases $(84 \%)$ at least two of the malignancyrelated parameters were easily recognizable together with reticulin framework abnormalities, whereas in only a minority of cases did the definition of malignancy rely on one single parameter (either necrosis, mitotic activity or venous invasion).

A step forward in our algorithmic approach was the identification of risk groups of adrenal cancer patients. In general terms, literature data on prognosis of adrenal cancer indicate a plethora of morphological, clinical or molecular features impacting on the clinical behaviour of this neoplasm, ${ }^{19,20,25-29}$ although some studies are biased by the sample size, unclear diagnostic criteria, and/or patients' selection. In agreement with literature data, in the present study multivariate survival analysis confirmed that diffuse architecture, stage and mitotic index represent the most relevant prognostic factors in adrenal cancer. Concerning mitotic index, the most significant prognostic cut off in our series was 9x50HPF (representing the median value), at variance with other Authors that used a level of 20x50HPF. ${ }^{12,20}$ However, irrespective of the cut off values employed, it is noteworthy that the vast majority of published series agrees that mitotic activity represents the tumor characteristic with the strongest impact on survival. 
The clinical impact of the prognostic factors pointed out in the present multivariate statistical model was further confirmed by survival analysis of the three risk groups identified according to the presence or absence of relevant adverse factors. The relevance of those findings, as compared to other studies in the literature ${ }^{30}$ is related to the large number of cases here analyzed, diagnosed and treated in one Institution. A validation set based on an external cohort of cases would be useful to strengthen the applicability of this algorithm.

In summary, in the present paper an easy-to-perform and highly specific and sensitive algorithm for the diagnosis of malignant adrenocortical tumors was designed, and a clinically relevant prognostic categorization of adrenal cancer patients was additionally provided. 


\section{References}

1. Fulmer BR. Diagnosis and management of adrenal cortical carcinoma. Curr. Urol. Rep. $2007 ; 8 ; 77-82$.

2. Kirschner LS. Emerging treatment strategies for adrenocortical carcinoma: a new hope. $J$. Clin. Endocrinol. Metab. 2006;91;14-21.

3. Paton BL, Novitsky YW, Zerey M, et al. Outcomes of adrenal cortical carcinoma in the United States. Surgery. 2006;140;914-920.

4. Terzolo M, Angeli A, Fassnacht M, et al. Adjuvant mitotane treatment for adrenocortical carcinoma. N. Engl. J. Med. 2007;356;2372-2380.

5. McNicol AM. Assessment of malignancy in adrenal cortical tumors. Endocr. Pathol. $2006 ; 17 ; 131-136$.

6. Volante M, Buttigliero C, Greco E, Berruti A, Papotti M. Pathological and molecular features of adrenocortical carcinoma: an update. J. Clin. Pathol. 2008;61;787-793.

7. Young WF Jr. Clinical practice. The incidentally discovered adrenal mass. N. Engl. J. Med. 2007;356;601-610.

8. Mantero F, Terzolo M, Arnaldi G, et al. A survey on adrenal incidentaloma in Italy. Study Group on Adrenal Tumors of the Italian Society of Endocrinology. J. Clin. Endocrinol. Metab. $2000 ; 85 ; 637-644$. 
9. Hough AJ, Hollifield JW, Page DL, et al. Prognostic factors in adrenal cortical tumors. A mathematical analysis of clinical and morphologic data. Am. J. Clin. Pathol. 1979;72;390-399.

10. van Slooten H, Schaberg A, Smeenk D, et al. Morphologic characteristics of benign and malignant adrenocortical tumors. Cancer. 1985;55;766-773.

11. Weiss LM. Comparative histologic study of 43 metastasizing and nonmetastasizing adrenocortical tumors. Am. J. Surg. Pathol. 1984;8;163-169.

12. Weiss LM, Medeiros LJ, Vickery AL Jr. Pathologic features of prognostic significance in adrenocortical carcinoma. Am. J. Surg. Pathol. 1989;13;202-206.

13. Aiba M, Fujibayashi M. Histopathological diagnosis and prognostic factors in adrenocortical carcinoma. Endocr. Pathol. 2005;16;13-22.

14. Aubert S, Wacrenier A, Leroy X, et al. Weiss system revisited: a clinicopathologic and immunohistochemical study of 49 adrenocortical tumors. Am. J. Surg. Pathol. 2002;26;1612-1619.

15. Stojadinovic A, Brennan MF, Hoos A, et al. Adrenocortical adenoma and carcinoma: histopathological and molecular comparative analysis. Mod. Pathol. 2003;16;742-751.

16. Wieneke JA, Thompson LD, Heffess CS. Adrenal cortical neoplasms in the pediatric population: a clinicopathologic and immunophenotypic analysis of 83 patients. Am. J. Surg. Pathol. $2003 ; 27 ; 867-881$. 
17. Blanes A, Diaz-Cano SJ. Histologic criteria for adrenocortical proliferative lesions: value of mitotic figure variability. Am. J. Clin. Pathol. 2007;127;398-408.

18. Terzolo M, Boccuzzi A, Bovio S, et al. Immunohistochemical assessment of Ki-67 in the differential diagnosis of adrenocortical tumors. Urology. 2001;57;176-182.

19. Gicquel C, Bertagna X, Gaston V, et al. Molecular markers and long-term recurrences in a large cohort of patients with sporadic adrenocortical tumors. Cancer. Res. 2001;61;6762-6767.

20. Assie G, Antoni G, Tissier F, et al. Prognostic parameters of metastatic adrenocortical carcinoma. J. Clin. Endocrinol. Metab. 2007;92;148-154.

21. Lack EE. Adrenal cortical carcinoma. In Lack EE, ed. AFIP Atlas of tumor pathology, series 4; Tumors of the adrenal glands and extraadrenal paraganglia. Washington DC: American Registry of Pathology Press; 2007;131-160.

22. Sullivan M, Boileau M, Hodges CV. Adrenal cortical carcinoma. J. Urol. 1978;120;660665.

23. Jarzembowski J, Lloyd R, McKeever P. Type IV collagen immunostaining is a simple, reliable diagnostic tool for distinguishing between adenomatous and normal pituitary glands. Arch. Pathol. Lab. Med. 2007;131;931-935.

24. Papotti M, Volante M, Bussolati G. Applied histochemistry in disorders of the endocrine system. In: Wick MR, ed. Diagnostic histochemistry. Cambridge: Cambridge University Press; 2008;280-306. 
25. Volante M, Sperone P, Bollito E, et al. Matrix metalloproteinase type 2 expression in malignant adrenocortical tumors: Diagnostic and prognostic significance in a series of 50 adrenocortical carcinomas. Mod. Pathol. 2006;19;1563-1569.

26. deFraipont F, El Atifi M, Cherradi N, et al. Gene expression profiling of human adrenocortical tumors using complementary deoxyribonucleic acid microarrays identifies several candidate genes as markers of malignancy. J. Clin. Endocrinol. Metab. 2005;90;1819-1829.

27. Evans HL, Vassilopoulou-Sellin R. Adrenal cortical neoplasms. A study of 56 cases. Am. J. Clin. Pathol. 1996;105;76-86.

28. Stojadinovic A, Ghossein RA, Hoos A, et al. Adrenocortical carcinoma: clinical, morphologic, and molecular characterization. J. Clin. Oncol. 2002;20;941-950.

29. Tauchmanovà L, Colao A, Marzano LA, et al. Andrenocortical carcinomas: twelve-year prospective experience. World. J. Surg. 2004;28;896-903.

30. Harrison LE, Gaudin PB, Brennan MF. Pathologic features of prognostic significance for adrenocortical carcinoma after curative resection. Arch. Surg. 1999;134;181-185. 


\section{Acknowledgements}

Work supported by grants from the Italian Ministry of University and Research (MIUR, Rome, ex $60 \%$ to MP, MT and MV) and from the Regione Piemonte (Ricerca Sanitaria Finalizzata 2007 to MV and MT)

We are grateful to Prof. G. Bussolati (University of Turin) for his helpful suggestions. 


\section{Figure legends}

Figure 1. Histochemical and immunohistochemical analysis of the reticulin framework in adrenocortical tumors. The presence of a disruption of the reticulin network was defined by the loss of continuity of reticular fibres in one high power field (400x) (a). The dotted line draws a virtual pathway connecting two opposite sides of a tumor field in malignant tumors. This connection was not possible in most adrenocortical adenomas, even in the case of large tumor size (b, adrenocortical adenoma, $9 \mathrm{~cm}$ in diameter), due to the integrity of the reticulin network. Conversely, it was a consistent finding in adrenocortical carcinomas (c) and was paralleled by the loss of basal membrane network (d, laminin immunohistochemistry), as compared to non-tumoral adrenal cortex (c and $\mathbf{d}$, insets)

(Original magnifications: a: 400x; b: 100x, c and d, including insets: 200x)

Figure 2. Simplified algorithm for the diagnosis and prognostic categorization of adrenocortical carcinomas (see text for details). ACA: adrenocortical adenoma. ACC: adrenocortical carcinoma.

Figure 3. Disease-free survival and overall survival curves of adrenocortical carcinomas segregated into three risk groups according to the proposed algorithm. 
Table 1. Clinico-pathological features of a series of 92 adrenocortical carcinomas and 47 adrenocortical adenomas with large size and/or Weiss score 1 or 2.

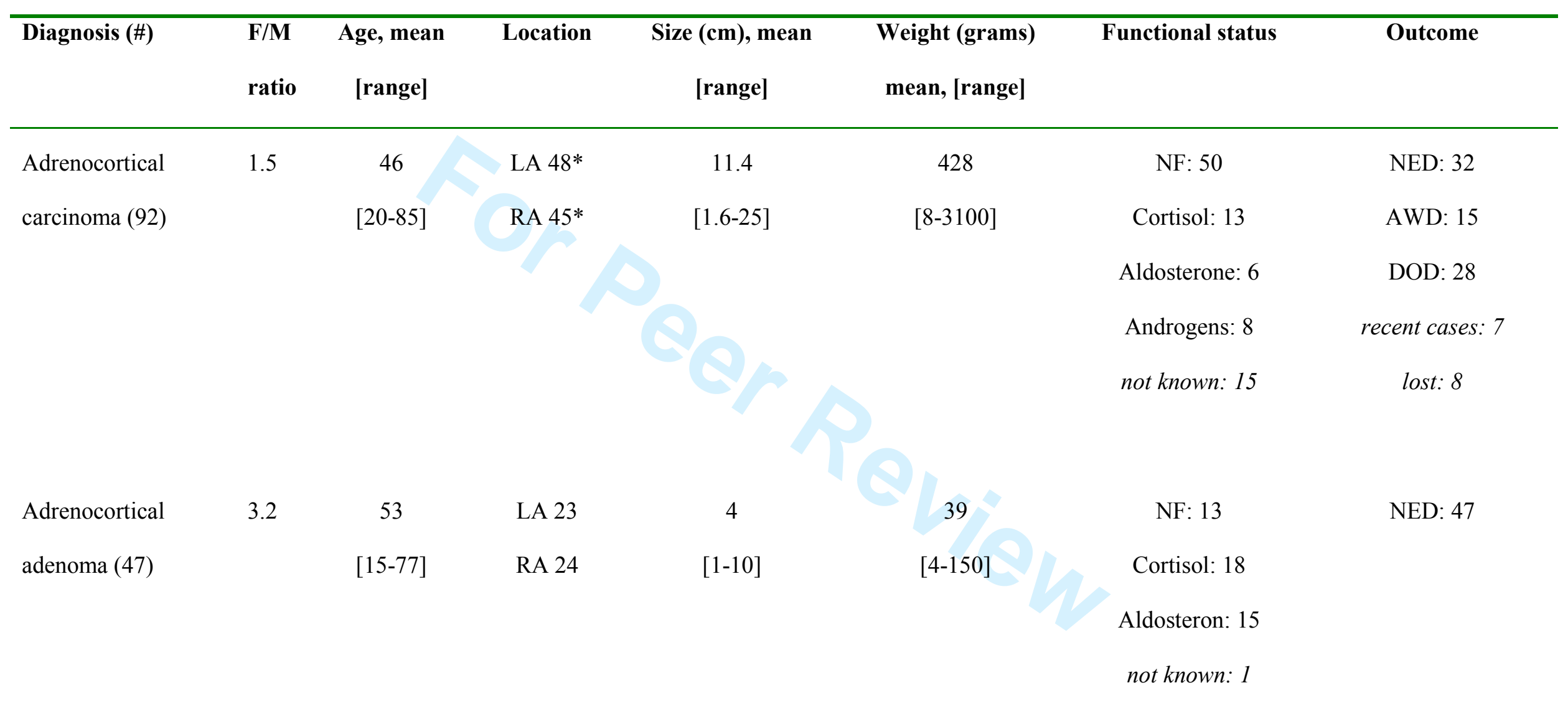

Legend. LA: left adrenal; RA: right adrenal; * one case had bilateral tumors, NF: not functioning; NED: no evidence of disease; AWD: alive with disease; DOD: died of disease. 
Table 2. Distribution of Weiss scoring system pathological parameters in 139 adrenocortical tumors, including 92 carcinomas and 47 adenomas.

\section{Frequency}

\begin{tabular}{lcccccc} 
Parameter & Weiss $<3$ & Weiss $\geq \mathbf{3}$ & SN & SP & PPV & NPV \\
\hline Mitoses $>\mathbf{5 x 5 0 H P F}$ & $0 / 47$ & $65 / 92$ & $71 \%$ & $100 \%$ & $100 \%$ & $64 \%$ \\
Atypical mitoses & $0 / 47$ & $31 / 92$ & $34 \%$ & $100 \%$ & $100 \%$ & $43 \%$ \\
Necrosis & $0 / 47$ & $77 / 92$ & $84 \%$ & $100 \%$ & $100 \%$ & $76 \%$ \\
Venous invasion & $0 / 47$ & $59 / 92$ & $64 \%$ & $100 \%$ & $100 \%$ & $59 \%$ \\
Sinusal invasion & $0 / 47$ & $52 / 92$ & $57 \%$ & $100 \%$ & $100 \%$ & $54 \%$ \\
Capsular invasion & $0 / 47$ & $50 / 92$ & $54 \%$ & $100 \%$ & $100 \%$ & $53 \%$ \\
Diffuse growth & $4 / 47$ & $73 / 92$ & $79 \%$ & $91 \%$ & $95 \%$ & $69 \%$ \\
Nuclear atypia & $9 / 47$ & $78 / 92$ & $85 \%$ & $81 \%$ & $90 \%$ & $73 \%$ \\
Clear cells <25\% & $6 / 47$ & $60 / 92$ & $65 \%$ & $87 \%$ & $91 \%$ & $56 \%$
\end{tabular}

SN: sensitivity; SP: specificity; PPV: positive predictive value; NPV: negative predictive value. 
Table 3. Multivariate analysis of disease-free and overall survival in adrenocortical carcinomas according to clinico-pathological parameters.

\section{Disease free survival}

Stage

$1-2$

3-4

Mitoses

$\leq 9$

$>9$

\section{Diffuse architecture}

Absent

Present

0.402

$0.196-0.822$

95\% Confidence Interval

$p$

Overall survival

Stage

$1-2$

3-4

2.349

$1.285-4.293$

0.006

Mitoses

$\leq 9$

$>9$

1.033

$1.012-1.053$

0.001

Diffuse architecture

Absent

Present

0.363

0.145-0.905

0.03 


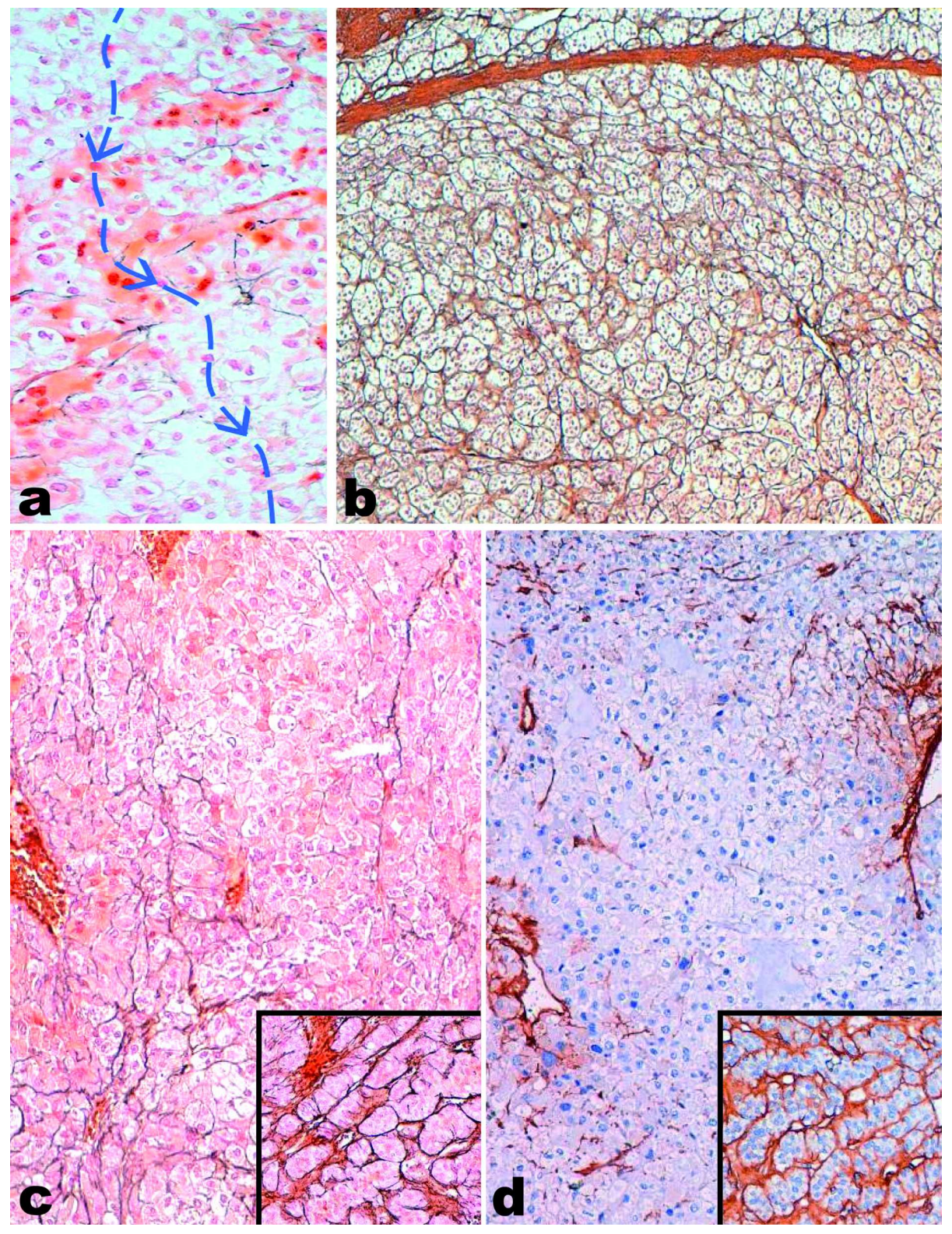

$160 \times 208 \mathrm{~mm}(300 \times 300$ DPI $)$ 


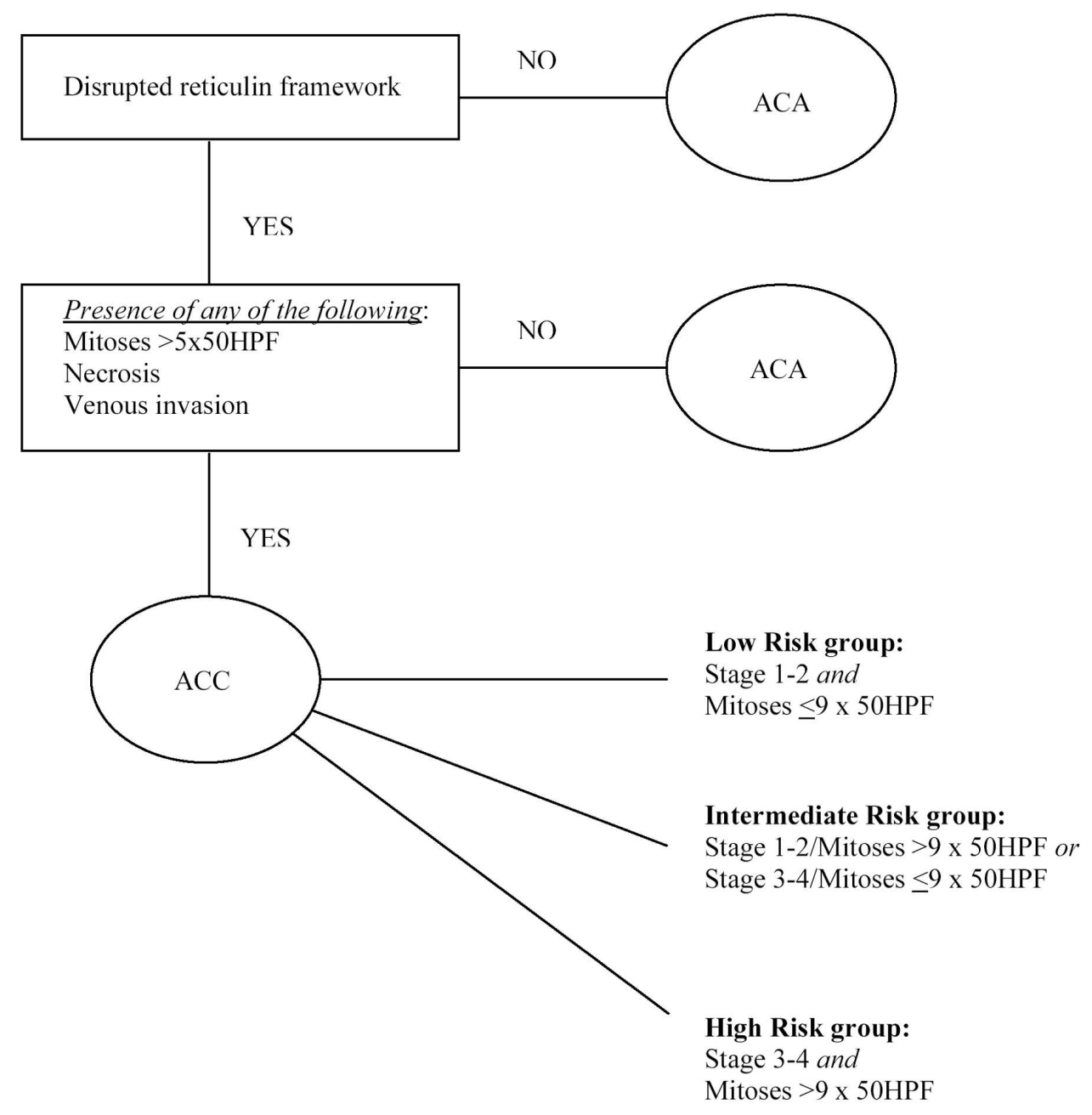

$160 \times 164 \mathrm{~mm}(300 \times 300$ DPI $)$ 

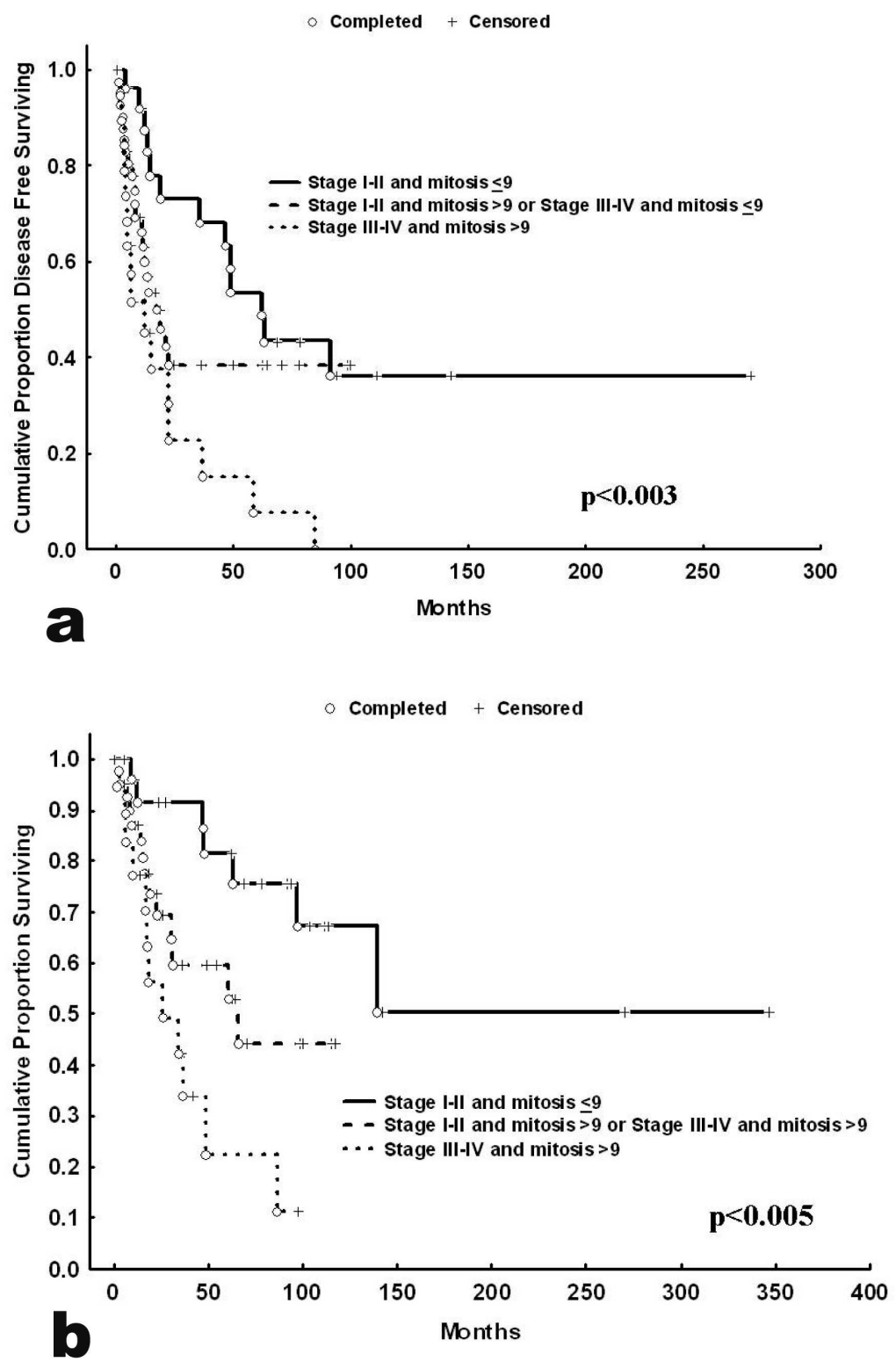

$160 \times 227 \mathrm{~mm}(300 \times 300 \mathrm{DPI})$ 